\title{
O Papel dos Inibidores do Fator de Necrose Tumoral no Tratamento da Artrite Idiopática Juvenil
}

\section{The Role of Tumor Necrosis Factor Inhibitors in the Treatment of Juvenile Idiopathic Arthritis}

\author{
Patrícia Martin $^{(1)}$, Ana Cristina de Medeiros ${ }^{(1)}$, Cláudia Goldenstein-Schainberg ${ }^{(2)}$
}

\section{RESUMO}

A artrite idiopática juvenil (AIJ) é uma doença crônica que pode evoluir para uma incapacidade permanente, necessitando muitas vezes de tratamento agressivo. Enquanto alguns pacientes não respondem ao metotrexato ou imunossupressores, outros não os toleram. Nestes casos, o uso dos inibidores de fator de necrose tumoral (TNF) ou anti-TNF é indicado. Conforme demonstrado por estudos multicêntricos e randomizados, o etanercepte apresenta eficácia no controle da doença, com efeito máximo nos primeiros três meses e até dois anos de tratamento contínuo. Não há publicação de estudos controlados com o infliximabe nem com o adalimumabe, mas seus efeitos parecem ser semelhantes aos do etanercepte. Estes agentes são relativamente seguros, podendo ser observadas complicações infecciosas, como a tuberculose e o herpes zoster. Raramente, podem ocorrer eventos auto-imunes, linfoproliferativos e sintomas gerais, como febre e cefaléia. No entanto, é importante salientar que apesar dos inibidores do TNF serem benéficos para muitas crianças com AIJ refratária, seus efeitos colaterais a longo prazo permanecem indefinidos, de forma que sua indicação deve ser criteriosa. Ademais, o alto custo destas drogas limita seu uso, especialmente em nosso país.

Palavras-chave: inibidores do fator de necrose tumoral, artrite idiopática juvenil, anti-TNF, artrite reumatóide juvenil.

\section{INTRODUÇÃO}

A artrite idiopática juvenil (AIJ) ou artrite reumatóide juvenil é a doença reumática crônica mais comum na criança na maioria dos países $^{(1)}$, sendo que 37 a $50 \%$ dos pacientes permanecem em atividade clínica ao atingirem a idade adulta ${ }^{(2,3,4)}$. Fantini et a ${ }^{(5)}$ mostraram que apenas 1/3 dos pacientes seguidos durante dez anos atingiram remissão. A duração cumulativa da atividade de doença é

\begin{abstract}
Juvenile Idiopathic Arthritis (JIA) is a chronic disease that may result in permanent disability, requiring sometimes aggressive treatment. While some patients may have an inadequate response to methotrexate and to other immunosuppressive drugs, others do not tolerate them. In these patients tumor necrosis factor inhibitors such as etanercept, infliximab and adalimumab are indicated. Multicentric and randomized studies have shown that etanercept is efficacious in disease control, reaching best effects within the first three months of therapy with sustained clinical improvement up to two years of continuous treatment. There are no published controlled studies involving children receiving infliximab and adalimumab, but their effects seem to be similar to that of etanercept. These drugs are relatively safe but it may be observed infectious complications, including tuberculosis and herpes zoster. Autoimmune events, lymphoproliferative disorders and other adverse reactions such as headache and abdominal pain barely occur. Nevertheless, it is important to emphasize that despite overall beneficial effects of TNF inhibitors, their long term effects remain undefined and therefore indications must be criterious. In addition, the high cost of these drugs limits their use, specially in our country.
\end{abstract}

Keywords: Tumor necrosis factor inhibitors, juvenile idiopathic arthritis, anti-TNF, juvenile rheumathoid arthritis.

preditiva da gravidade da limitação funcional ${ }^{(6)}$, já que sua persistência resulta em dor crônica e rigidez, dano articular irreversível, retardo do crescimento e limitação funcional importante ${ }^{(7)}$, justificando tratamento agressivo precoce.

Os antiinflamatórios não-esteroidais (AINEs) ainda constituem a terapia de primeira linha para AIJ, cujo efeito deve ser observado por volta de um mês após seu início ${ }^{(1)}$. Aproximadamente um terço dos pacientes podem ter a doença controlada com AINEs aliados a programas adequados

Trabalho realizado pela Disciplina de Reumatologia da Faculdade de Medicina da Universidade de São Paulo (USP). Recebido em 14/3/2005. Aprovado, após revisão, em 04/01/06.

1. Médica Residente de Reumatologia do Hospital das Clínicas da Faculdade de Medicina da USP.

2. Doutora em Medicina, Professora Assistente da Disciplina de Reumatologia da Faculdade de Medicina da USP.

Endereço para correspondência: Patrícia Martin, Av. Dr Arnaldo, 455, sala 3133, São Paulo, CEP 01246-930, SP, Brasil, telefone: (11)3066-7490,

e-mail: patriciamartin2004@ig.com.br 
de fisioterapia e terapia ocupacional, enquanto os demais são candidatos a terapêuticas com drogas modificadoras de doença ou anti-reumáticas ${ }^{(8)}$. O metotrexato (MTX) é o medicamento de segunda linha de escolha para pacientes com quadro ativo persistente ${ }^{(9,10)}$. A dose inicial de 10 $\mathrm{mg} / \mathrm{m}^{2} /$ semana pode ser progressivamente aumentada na ausência de resposta ${ }^{(11,12)}$ e pode ser administrada de forma parenteral até $15 \mathrm{mg} / \mathrm{m}^{2} /$ semana ou $1 \mathrm{mg} / \mathrm{kg} /$ dose $^{(13)}$. Seu efeito terapêutico máximo é observado em nove meses a um ano de uso contínuo. ${ }^{(13)}$

Antimaláricos (cloroquina e hidroxicloroquina) também têm sido utilizados na AIJ há mais de 40 anos devido ao seu efeito imunomodulador ${ }^{(14,15)}$, apesar de estudo recente não ter demonstrado superioridade da hidroxicloroquina nem da penicilamina sobre o placebo ${ }^{(16)}$.

Dentre outras drogas que podem ser prescritas no tratamento da AIJ, destacamos sulfassalazina ${ }^{(17)}$, azatioprina ${ }^{(18,}$ ${ }^{19)}$, ciclosporina $^{(20,21)}$, leflunomida ${ }^{(22)}$ e ciclofosfamida ${ }^{(23,24)}$, todas com indicação, eficácia e efeitos colaterais variáveis. Pode-se ainda usar clorambucil, cautelosamente, em casos de amiloidose secundária ${ }^{(25,26)}$ e na uveíte refratária ${ }^{(27)}$. Atualmente, não há dados que justifiquem o uso de gamaglobulina na $\mathrm{AIJ}^{(2,14)}$, exceto em casos de doença sistêmica não-responsiva ${ }^{(28)}$.

\section{AGENTES BIOLÓGICOS NO TRATAMENTO DA AIJ}

Recentemente, agentes biológicos têm sido utilizados no arsenal terapêutico das doenças auto-imunes. Na AIJ refratária, os inibidores de fator de necrose tumoral alfa (TNF) parecem promissores ${ }^{(2)}$, já que o TNF é uma citocina pró-inflamatória com papel relevante na patogênese da artrite reumatóide ${ }^{(29-35)} \mathrm{e}$ da $\mathrm{AIJ}{ }^{(36,37)}$. O TNF está elevado no líquido sinovial ${ }^{(36)}$ e no soro $^{(37)}$ de crianças com AIJ e seu valor sérico está relacionado com o grau de atividade de doença ${ }^{(37)}$. Grom et a ${ }^{(38)}$ encontraram TNF alfa em 34 de 40 amostras de tecido sinovial de crianças com AIJ e sua expressão estava relacionada ao grau de infiltração inflamatória nos tecidos. Crianças com espondiloartropatia juvenil apresentavam expressão proeminente de TNF alfa. Já o TNF beta foi encontrado no tecido sinovial de 22 das 26 amostras de pacientes com AIJ e de 4 das 6 amostras de pacientes com espondiloartropatia juvenil.

Atualmente, existem no mercado três agentes bloqueadores da ação do TNF: etanercepte, infliximabe e adalimumabe. O etanercepte é um análogo do receptor do fator de necrose tumoral e consiste em dois receptores solúveis de TNF combinados à porção $\mathrm{Fc}$ da imunoglobulina $\mathrm{G}$ (IgG) humana ${ }^{(39)}$ de modo que se liga ao TNF e impede sua interação com as células. Desta forma, o etanercepte não age diretamente no TNF, mas, sim, bloqueia sua ação. Já o infliximabe é um anticorpo monoclonal quimérico dirigido contra o fator de necrose tumoral que se liga ao TNF solúvel e ao TNF ligado à membrana celular ${ }^{(40)}$, enquanto que o adalimumabe oferece a vantagem de ser um anticorpo monoclonal totalmente humanizado dirigido contra o $\mathrm{TNF}^{(40)}$.

Dentre estes três agentes inibidores de TNF, apenas o etanercepte foi aprovado pela Food and Drugs Administration (FDA) para uso em crianças, particularmente no tratamento da AIJ moderada à grave e resistente a outras drogas ${ }^{(2)}$. Não há estudos controlados publicados que utilizem infliximabe nem o adalimumabe na faixa etária pediátrica. Quanto à posologia a ser utilizada neste grupo de pacientes estão estabelecidas as seguintes doses: etanercepte $(0,4 \mathrm{mg} / \mathrm{kg}$ subcutâneo duas vezes por semana), infliximabe ( 3 a 5 $\mathrm{mg} / \mathrm{kg}$ nas semanas 0,2 e 6 e depois a cada 8 semanas em infusão intravenosa) e adalimumabe $\left(24 \mathrm{mg} / \mathrm{m}^{2} \mathrm{de}\right.$ superfície corporal).

Um dos fatores limitantes para o uso dos inibidores de TNF, especialmente em nosso meio é o alto custo desta forma de terapia que onera não apenas o Serviço Público e as cidades onde não há Serviço de Reumatologia estruturado, mas também os convênios que não liberam este tipo de tratamento e até mesmo o paciente particular que necessita destas drogas.

\section{ETANERCEPTE}

Dois estudos prospectivos e multicêntricos demonstraram a eficácia e segurança do etanercepte em crianças com AIJ ${ }^{(8,40)}$. Nos Estados Unidos, Lovell et al ${ }^{8)}$ avaliaram o seu uso em 69 pacientes (4 a 17 anos de idade) com doença poliarticular resistentes ou intolerantes ao metotrexato. $\mathrm{O}$ estudo consistiu em duas fases: na primeira, de forma aberta, não-comparativa, todos receberam etanercepte por três meses, havendo resposta favorável em 51 (74\%) indivíduos. Na segunda fase, as 51 crianças respondedoras foram randomizadas em dois grupos: 26 receberam placebo e 25 etanercepte, sendo que o grupo tratado com a droga ativa teve menos dor e edema nas articulações, menor incidência de atividade de doença e recidivas menos freqüentes que o grupo placebo. Após 28 dias, $81 \%$ dos pacientes do grupo 
placebo estavam em atividade, enquanto apenas $28 \%$ dos que receberam etanercepte apresentaram manifestações articulares. Cinqüenta e oito pacientes continuaram o estudo recebendo etanercepte e após 2 anos, 43 crianças apresentaram resposta persistente com o uso contínuo da medicação. $^{(40)}$

Outro estudo com o etanercepte foi conduzido por Quartier et $\mathrm{al}^{(41)} \mathrm{em}$ centros franceses e englobou 61 crianças com AIJ de início sistêmico, poliarticular ou oligoarticular (média de idade $=12$ anos) que não obtiveram resposta prévia ou que apresentaram efeitos colaterais com outras drogas, tais como metotrexato, ciclosporina, D-penicilamina, hidroxicloroquina, sulfassalazina, azatioprina, pulsoterapia com metilprednisolona, imunoglobulina ou ciclofosfamida intravenosas. O seguimento foi de 18 meses em média e a eficácia máxima da droga foi vista três meses após o início do etanercepte; chama atenção o fato de que os pacientes que não atingiram resposta favorável até este período não apresentaram melhora nos meses subseqüentes. Após 1 ano de tratamento, 9 pacientes (14\%) tiveram reativação da doença e, dentre as 24 crianças que foram tratadas por mais de 15 meses, $19(79,1 \%)$ obtiveram melhora de 30 a 70\% em relação ao nível basal. Quanto à forma de apresentação, a AIJ de início sistêmico teve maiores índices de falha terapêutica, indicando que talvez estes pacientes apresentem menor resposta ao tratamento com anti-TNF, provavelmente devido aos altos níveis de interleucina- 6 circulante nestas crianças. Portanto, neste subgrupo de pacientes, o uso de agentes anti-IL-6 específicos parece mais adequado e tem levado a uma melhor resposta terapêutica. ${ }^{(42,43)}$

É importante salientar que pacientes que não respondem às doses convencionais de etanercepte parecem não se beneficiar com incrementos nas dosagens, conforme demonstrado por Takei et $a^{(44)}$ ao estudar retrospectivamente 8 crianças com idade média de 8,4 anos que não melhoraram com as doses preconizadas de $0,4 \mathrm{mg} / \mathrm{kg}$ por via subcutânea (SC) 2 vezes por semana. De fato, apenas 2 dos 8 pacientes (25\%) obtiveram melhora clínica e laboratorial com doses médias de $1,1 \mathrm{mg} / \mathrm{kg}$ (dose máxima de $25 \mathrm{mg}$ SC 2 vezes/semana).

Quanto ao controle da uveíte crônica refratária, ainda não está definido o papel do etanercepte, uma vez que três estudos mostraram resultados conflitantes. No primeiro deles, prospectivo, não-controlado, envolvendo 10 crianças (média de idade de 7,5 anos) não-responsivas ao tratamento tópico, ao metotrexato nem à ciclosporina ${ }^{(45)}$ houve resposta favorável ao uso do etanercepte. O segundo estudo retrospectivo, e abrangendo 16 pacientes bastante heterogêneos com uveíte e ampla variação de idade ( 7 a 78 anos), mostrou controle apenas da manifestação articular, mas não da doença ocular ${ }^{(46)}$. Finalmente, $\mathrm{o}$ terceiro estudo randomizado duplo-cego e controlado com placebo não mostrou benefício com o uso de etanercepte, o que poderia ser explicado pelo pequeno número de pacientes envolvidos (cinco recebendo placebo comparados com sete utilizando etanercepte $)^{(47) .}$

Há, ainda, relato de um menino de sete anos de idade com síndrome de ativação macrofágica que não apresentou resposta à corticoterapia, mas que melhorou em 24 horas após uso de etanercepte, permanecendo assintomático após 11 semanas de terapêutica e sem recidiva após a retirada do medicamento $^{(48)}$.

Ensaios clínicos controlados em adultos com artrite reumatóide ${ }^{(49)}$ e estudos observacionais envolvendo crianças com AIJ ${ }^{(50)}$ comparando a associação do etanercepte com metotrexato ou com placebo sugerem efeito aditivo e toxicidade tolerável com o uso concomitante das duas drogas. Apesar de não haver estudo controlado comparando metotrexato com etanercepte na AIJ, adultos com artrite reumatóide precoce tratados com etanercepte tiveram menor progressão radiológica e resposta clínica mais rápida do que aqueles tratados apenas com metotrexato. Após 2 anos de seguimento, os pacientes que receberam etanercepte apresentaram menos erosões e menos atividade de doença. ${ }^{(51,52)}$

\section{INFLIXIMABE}

Gerloni et al ${ }^{(53)}$ tratou 20 pacientes do sexo feminino com AIJ refratária com duração média de doença de 17 anos (5 a 31 anos), obtendo rápida melhora em todas, sendo que 8 mantiveram boa resposta após 12 meses de tratamento. Lahdene e Honkanen compararam oito crianças que receberam infliximabe com sete tratadas com etanercepte e ambos os grupos alcançaram excelentes respostas clínicas. Apenas duas pacientes do grupo tratado com infliximabe pararam a medicação por efeitos colaterais (febre e pancitopenia em uma e alopecia e surgimento de anti-DNA em outra) $)^{(54)}$. Posteriormente, estes mesmos autores observaram rápida melhora e redução persistente da atividade da doença com ambos agentes ao analisarem 24 crianças em estudo aberto, não-randomizado e prospectivo em que 14 usaram infliximabe e 10 utilizaram etanercepte. Foi observado que cinco crianças do grupo infliximabe interromperam o tratamento por falta de 
eficácia ou por efeitos colaterais, enquanto apenas uma criança do grupo etanercepte parou a medicação devido à má aderência. ${ }^{(55)}$

\section{ADALIMUMABE}

Apesar da vasta e favorável experiência em adultos com artrite reumatóide ${ }^{(56,57)}$, há apenas um estudo em andamento englobando crianças, cujos resultados preliminares mostraram boa resposta já na segunda semana de tratamento, que se manteve até 16 semanas de uso contínuo da droga. A fase inicial do estudo foi aberta com duração de 16 semanas e envolveu 155 crianças com idade entre 4 e 17 anos. A dose utilizada foi $24 \mathrm{mg} / \mathrm{m} 2$ de superfície corporal a cada 15 dias. É importante salientar que foi placebo controlado, multicêntrico e randomizado. Ademais, foi permitido uso concomitante de metotrexato, o que ocorreu em $52 \%$ dos $\operatorname{casos}^{(58)}$.

\section{EFEITOS ADVERSOS DOS INIBIDORES DE TNF}

Os efeitos adversos observados com o uso de agentes anti-TNF incluem complicações alérgicas durante ou após a infusão, infecções e eventos auto-imunes ${ }^{(59)}$. A freqüência destes efeitos parece ser baixa em adultos de modo que a terapia com infliximabe e etanercepte parece ser segura ${ }^{(59-61)}$. Existem, porém, relatos esporádicos de efeitos colaterais graves e ainda não está definido o que pode resultar da inibição do TNF por longos períodos de tempo. Desta forma, já que crianças com AIJ têm o potencial para usar estes medicamentos por longos períodos de tempo, sua indicação deve ser bastante cautelosa e criteriosa ${ }^{(59)}$.

Dentre as complicações auto-imunes foram descritas síndromes desmielinizantes do sistema nervoso central $(\mathrm{SNC})^{(62)} \mathrm{e}$ diabete tipo $\mathrm{I}^{(40,63)} \mathrm{em}$ crianças usando etanercepte. Em adultos, casos de lúpus eritematoso sistêmico foram relatados após uso de inibidores de TNF. ${ }^{(64-66)}$

Em relação às neoplasias, desde a introdução dos inibidores do TNF, a possibilidade destas drogas aumentarem o risco de malignidade vem sendo questionada, uma vez que entre março de 1999 e dezembro de 2000 foram relatados ao FDA 26 novos casos de pacientes que tiveram doenças linfoproliferativas após o uso desta forma de terapia ${ }^{(67)}$. Porém, Wolfe e Michaud ${ }^{(68)}$ ao estudarem prospectivamente 18.572 adultos com artrite reumatóide durante 3 anos não puderam estabelecer relação causal entre uso de biológicos e desenvolvimento de doenças linfoproliferativas. De for-
TABELA 1

Eventos ADVERSOS MENOS COMUNS EM CRIANÇAS QUE USARAM AGENTES ANTI-TNF

\begin{tabular}{|c|c|c|}
\hline AUTOR & DROGA & EFEITO ADVERSO \\
\hline Lovell(40) & etanercepte & $\begin{array}{l}\text { cefaléia, dor abdominal, rinite, } \\
\text { náusea, febre, eritema, depressão, } \\
\text { reações no local da injeção }\end{array}$ \\
\hline Quartier(41) & etanercepte & $\begin{array}{c}\text { manifestações psiquiátricas } \\
\text { graves, pancitopenia, eritema } \\
\text { vasculítico, ganho de peso ( } 6 \\
\text { a } 20 \mathrm{Kg} \text { ), neuropatia óptica } \\
\text { retrobulbar, uveíte }\end{array}$ \\
\hline $\operatorname{Ramanan}^{(73)}$ & etanercepte & síndrome de ativação macrofágica \\
\hline $\begin{array}{l}\text { Kaipiaienen- } \\
\text { Seppann }{ }^{(74)}\end{array}$ & $\begin{array}{l}\text { etanercepte e } \\
\text { infliximabe }\end{array}$ & uveíte recorrente \\
\hline Foeldvari $i^{(75)}$ & $\begin{array}{l}\text { etanercepte e } \\
\text { infliximabe }\end{array}$ & colecistite aguda não-obstrutiva \\
\hline Tutar ${ }^{(76)}$ & infliximabe & $\begin{array}{c}\text { eritema maculopapular } \\
\text { urticariforme }\end{array}$ \\
\hline Lovell(58) & adalimumabe & gastrite aguda \\
\hline
\end{tabular}

ma semelhante, há relatos isolados de carcinoma de células escamosas $^{(69-71)}$ e leucemia mielóide aguda ${ }^{(72,73)}$ em adultos que usaram inibidores de TNF, mas é importante ressaltar que na faixa etária pediátrica, até o momento, nenhum caso de neoplasia foi relatado ${ }^{(59)}$.

Outros efeitos adversos menos comuns na faixa etária pediátrica podem ser observados na Tabela 1 .

Em crianças, as complicações infecciosas associadas ao uso de inibidores de TNF variam de quadros leves a potencialmente fatais (Tabela 2). Sete ${ }^{(40,78)}$ apresentaram herpes

TABELA 2

COMPLICAÇÕES INFECCIOSAS DOS ANTI-TNF EM CRIANÇAS

\begin{tabular}{|c|c|c|c|c|}
\hline Autor & $\mathrm{n}$ & Tempo & Droga & Complicações infecciosas \\
\hline Lovell ${ }^{(40)}$ & 58 & 2 anos & etanercepte & $\begin{array}{c}\text { faringite, infecções } \\
\text { de pele, gripe, otite e } \\
\text { conjuntivite, herpes zoster, } \\
\text { apendicite/peritonite, } \\
\text { infecção de partes moles, } \\
\text { infecção pós-operatória, } \\
\text { sepse, infecções urinárias e } \\
\text { de vias aéreas superiores }\end{array}$ \\
\hline Quartier ${ }^{(41)}$ & 61 & 18 meses & etanercepte & apendicite \\
\hline Sthepens* ${ }^{(77)}$ & 82 & 26 meses & infliximabe & $\begin{array}{l}\text { herpes zoster, meningite } \\
\text { por Lysteria }\end{array}$ \\
\hline Lovell(58) & 155 & 16 semanas & adalimumabe & herpes genital, pneumonia \\
\hline
\end{tabular}

* pacientes com doença de Chron. 
zoster, sendo que um menino evoluiu com meningite asséptica. É importante salientar que elas não haviam sido vacinadas contra varicela zoster; a infecção ocorreu entre 5 e 15 meses após o início do tratamento com etanercepte. Desta forma, recomenda-se que toda criança em uso de agentes anti-TNF expostas à varicela sem história prévia de doença seja tratada com imunoglobulinas e aciclovir ao primeiro sinal desta infecção. ${ }^{(40)}$

\section{TUBERCULOSE E INIBIDORES DE TNF}

A tuberculose (TB) tem sido observada em adultos em uso de agentes anti- $\operatorname{TNF}^{(79,80)}$. A redução na atividade do fator de necrose tumoral durante o tratamento com inibidores de TNF alfa dificulta a compartimentalização da micobactéria por inibir a formação do granuloma, levando à reativação da TB latente ${ }^{(81)}$. Em crianças em uso de agentes anti-TNF, até o momento, há apenas dois relatos de $\mathrm{TB}^{(59}$, ${ }^{82)}$. No entanto, com o aumento do uso destes agentes terapêuticos, a probabilidade deste tipo de complicação poderá crescer se cuidados na seleção de crianças e exclusão de doença latente não forem avaliados de forma criteriosa e adequada, especialmente em nosso país, onde a TB ainda é um problema de saúde pública. ${ }^{(83)}$

Neste sentido, doença latente deve ser sempre pesquisada em crianças, o que pode ser difícil em nosso meio, uma vez que a vacina com BCG é obrigatória a todos os recémnascidos e a imunidade conferida pelo BCG é dependente do linfócito de memória, podendo se prolongar por 10 a $15 \operatorname{anos}^{(84)}$, dificultando a interpretação do teste de PPD.

\section{REFERÊNCIAS}

1. Lovell DJ: Juvenile Rheumatoid arthritis and juvenile spondyloarthropathies. Primer on the rheumatic diseases, 12 ed, Atlanta, Arthritis Foudation, 2001.

2. Schneider R, Passo MH: Juvenile rheumatoid arthritis. Rheum Dis Clin N Am 28: 503-30, 2002.

3. Zak M, Peterssen, FK: Juvenile rheumatoid arthritis into adulthood: a long term follow up study. Rheumatology 39: 198-204, 2000.

4. Foster HE, Marshall N, Myers A, Dunkley P, Griffiths ID: Outcome in adults with juvenile idiopathic arthritis: a quality of life study. Arthritis Rheum 48: 767-75, 2003.

5. Fantini F, Gerloni V, Gattinara M, Cimaz R, Arnoldi C, Lupi E: Remission in juvenile chronic arthritis: a cohort study of 683 consecutive cases with a mean 10 year follow up. J Rheumatol 3: 579-84, 2003.

6. Lomater $\mathrm{C}$, Gerloni V, Gattinara $\mathrm{M}$ et al: Systemic onset juvenile idiopathic arthritis: a retrospective study of 80 consecutive patients followed for 10 years. J Rheumatol 27: 491-6, 2000.
Entretanto, dois estudos brasileiros mostraram que todo PPD superior a $10 \mathrm{~mm}$ não está relacionado à vacinação, mas sim à exposição e contato com adultos com TB. ${ }^{(85,86)}$

Portanto, antes de iniciar a terapêutica com inibidores de TNF, recomenda-se sempre investigar TB latente. Se o PPD for maior ou igual a $5 \mathrm{~mm}$ com raio $\mathrm{X}$ de tórax normal ou compatível com seqüela ${ }^{(84)}$, a quimioprofilaxia específica está indicada e a droga preconizada é a isoniazida na dose de $10 \mathrm{mg} / \mathrm{kg} /$ dia por via oral, não excedendo $300 \mathrm{mg}$, durante 6 meses consecutivos ${ }^{(84)}$.

\section{CONCLUSÕES}

Pretendemos com esta revisão ressaltar que a terapêutica da AIJ vem evoluindo rapidamente. Muitos pacientes, antes resistentes ou intolerantes às drogas modificadoras de doença e imunossupressores têm se beneficiado com o uso de inibidores de TNF, que têm potencial para melhorar a evolução e o prognóstico da doença, além da qualidade de vida destas crianças. Estes medicamentos são relativamente seguros e as infecções são as complicações mais freqüentes. A possibilidade de TB latente deve sempre ser pesquisada e, se necessário, deve ser iniciada quimioprofilaxia com isoniazida. Ressaltamos, ainda, que o uso destes agentes deve ser criterioso e reservado para doença refratária, já que crianças com AIJ são candidatas a sua utilização por longos períodos de tempo. Finalmente, esta terapêutica não é curativa para a AIJ, seus efeitos a longo prazo ainda não estão definitivamente estabelecidos e seu custo extremamente elevado permanece um fator limitante para sua ampla utilização no tratamento de crianças com AIJ em nosso meio.

7. Wilkinson N, Jackson G, Gardner-Medwin J: Biologic therapies for juvenile arthritis. Arch Dis Child 88: 186-91, 2003.

8. Lovell DJ, Giannini EH, Reiff A et al: Etanercept in children with poliarticular juvenile rheumatoid arthritis. N Engl J Med 342: 763-69, 2000.

9. Giannini EH, Brewer EJ, Kuzmina $\mathrm{N}$ et al: Methotrexate in resistant juvenile rheumatoid arthritis: results of de USA-USRR double blind, placebo controlled trial. N Eng J Med 326: 1043$49,1992$.

10. Gianinni EH, Cassidy JT, Brewer EJ, Shaikov A, Kuzmina N: Comparative efficacy and safety of advanced drug therapy in children with juvenile rheumatoid arthritis. Semin Arthritis Rheum 23: 34-46, 1993.

11. Wallace CA, Sherry DD: Preliminary report of higher dose methotrexate treatment in juvenile rheumatoid arthritis. J Rheumatol 19: 1604-07, 1992.

12. Reiff A, Shaham B, Wood BP: High dose methotrexate in the treatment of refractory juvenile rheumatoid arthritis. Clin Exp Rheumatol 13: 113-8, 1995.

13. Ruperto N, Murray KJ, Gerloni V, Wulffrat N, de Oliveira SKF, 
Falcini $\mathrm{F}$ et al: A randomized trial of parenteral methotrexate comparing an intermediate dose with a higher dose in children with juvenile idiopathic arthritis who failed to respond to standard doses of methotrexate. Arthritis Rheum 50: 21912201, 2004.

14. Prier AM, Quartier P: Comparative tolerability of treatments for juvenile idiopathic arthritis. Bio Drugs 14: 159-83, 2000.

15. Kvien TK, Hoyeraal HM, Sandstad B: Slow acting antirheumatic drugs in patients with juvenile rheumatoid arthritis evaluated in a randomized, parallel 50 week clinical trial. J Rheumatol 12: 533-9, 1985.

16. Brewer EJ, Giannini EH, Kuzmina N, Alekseev L: Penicillamine and hydroxychloroquine in the treatment of severe juvenile rheumatoid arthritis. Results of the USA-USSR double blind placebo controlled trial. N Engl J Med 314: 1269-76, 1986.

17. Brooks CD: Sulfasalazine for the management of juvenile rheumatoid arthritis. J Rheumatol 28: 845-53, 2001.

18. Kveien TK, Hoyeraal HM, Sandstad B: Azathioprine versus placebo in patients with juvenile rheumatoid arthritis: a single center double blind comparative study. J Rheumatol 13: 118$23,1986$.

19. Savolainen HA, Kautiainen H, Isomaki H, Aho K, Verronen P: Azathioprine in patients with juvenile chronic arthritis: a long term follow up study. J Rheumatol 24: 2444-50, 1997.

20. Gerloni V, Cimaz R, Gattinara M, Arnoldi C, Pontikaki I, Fantini F: Efficacy and safety profile of cyclosporine $\mathrm{A}$ in the treatment of juvenile rheumatoid (idiopathic) arthritis: results of a 10 years prospective study. Rheumatology 40: 907-13, 2001.

21. Reiff A, Rawlings DJ, Shaham B, Franke E, Richardson L, Szer IS, Bernstein BH: Preliminary evidence for cyclosporine A as an alternative treatment of recalcitrant juvenile rheumatoid arthritis and juvenile polymyositis. J Rheumatol 24: 2436-43, 1997.

22. Silverman E, Mouy R, Spiegel L, Jung LK, Saurenmann RK, Lahdenne P, Horneff $\mathrm{G}$ et al: Leflunomide or methotrexate for juvenile rheumatoid arthritis. N Engl J Med 352: 1655-66, 2005.

23. Shaikov AV, Maximov AA, Speranski AI: Repetitive use of pulse therapy with methylprednisolone and cyclophosphamide in addition to oral methotrexate in children with systemic juvenile rheumatoid arthritis: preliminary results of a long term study. J Rheumatol 19: 612-6, 1992.

24. Wallace CA, Sherry DD. Trial of intravenous pulse cyclophosphamide and methylprednisolone in the treatment of systemic onset rheumatoid arthritis. Arthritis Rheum 40: 1852-5, 1997.

25. Ansell BM: Chorambucil therapy in juvenile chronic arthritis (juvenile idiopathic arthritis). J Rheumatol: 26: 765-6, 1999.

26. Savolainen HA: Chlorambucil in severe juvenile chronic arthritis: longterm follow up with special reference to amyloidosis. J Rheumatol 26: 898-906, 1999.

27. Miserochi E, Baltazis S, Ekong E, Roque M, Foster S: Efficacy and safety of chlorambucil in intractable non infectious uveitis. The Massachusetts eye and ear infirmary experience. Ophthalomology 109: 137-142, 2002.

28. Silverman ED, Laxer RM, Greenwald M, Gelfand E, Shore A, Stein LD, Roifman CM: Intravenous gamma globulin therapy in systemic juvenile rheumatoid arthritis. Arthritis Rheum 33: 1015-22, 1990.
29. Dayer JM, Beutler B, Cerami A: Cachetin/tumor necrosis factor stimulates collagenase and prostaglandin E2 production by human synovial cells and dermal fibroblasts. J Exp Med 162: 2163-8, 1985.

30. Bertolini DR, Nedwin GE, Bringman TS, Smith DD, Mundy GRY: Stimulation of bone resorption and inhibition of bone formation in vitro by human tumor necrosis factors. Nature 319 : 516-8, 1986.

31. Saklatvala J: Tumor necrosis factor alpha stimulates resorption and inhibits synthesis of proteoglycan in cartilage. Nature 322: 547-9, 1986.

32. von Asmuth E JU, van der Linden CJ, Leeuwenberg JFM, Buurman WA: Involvement of the CDIlb/CD18 integrin, but not of the endothelial cell adhesion molecules ELAM-1 and ICAM-1 in tumor necrosis factor alpha induced neutrophil toxicity. J Immunol 147: 3869-75, 1991.

33. Beckham JC, Caldwell DS, Peterson BL et al: Disease severity in rheumatoid arthritis: relationships of plasma tumor necrosis factor alpha, soluble interlukin 2 receptor, soluble CD4/CD8 ratio, neopterin, and fibrin $\mathrm{D}$ dimer to traditional functional and severity measures. J clin Immunol 12: 353-61, 1992.

34. Brennan FM, Chandry D, Jackson A, Maini R, Feldman N: Inhibitory effect of TNF alpha antibodies on synovil cell interleukin-1 production in rheumatoid arthritis. Lancet 2: 2447, 1989.

35. Saez Lorens X, Jafari HS, Olsen KD, Nariuchi H, Hansen EJ, Mc Craken GH Jr: Induction of supurative arthritis in rabbits by Haemophilus endotoxin, tumor necrosis factor alpha and interleukin 1 beta. J Infec Dis 163: 1267-72, 1991.

36. Eberhard BA, Laxer RM, Anderson U, Silvermann ED: Local synthesis of both macrophage and T cells cytokines by sinovial fluid cells from children with juvenile rheumatoid arthritis. Clin Exper Immunol 96: 260-6, 1994.

37. Mangge H, Kenzian H, Gallistl S et al: Serum cytokines in juvenile rheumatoid arthritis. Correlation with conventional inflammation parameters and clinical subtypes. Arthritis Rheum 38: 211-20, 1995.

38. Grom AA, Murray KJ, Luyrink L et al: Patterns of expression of tumor necrosis factor alfa, tumor necrosis factor beta, and their receptors in sinovia of patients with juvenile rheumatoid arthritis and juvenile spondiloarthropathy. Arthritis Rheum, 39: 1703 $-10,1996$.

39. Olsen NJ, Stein MC: New drugs for Rheumatoid Arthritis. N Eng J Med, 350: 2167-2179, 2004.

40. Lovell DJ, Giannini HE, Reiff A et al: Long term efficacy and safety of etanercept in children with poliarticular course juvenile rheumatoid arthritis: interim results from an ongoing multicenter, open label, extended treatment trial. Arthritis Rheum 48: 218-26, 2003.

41. Quartier P, Taupin P, Bourdeaut F et al: Efficacy of etanercept for the treatment of juvenile idiopathic arthritis according to the Onset Type. Arthritis Rheum 48: 1093-101, 2003.

42. de Benedetti F, Martini A: Targeting the Interleukin-6 receptor: a new treatment for Systemic idiopathic arthritis? Arthritis and Rheum 52: 687-93, 2005.

43. Yokoda S, Miyamae T, Imagawa T: Therapeutic efficacy of humanized recombinant anti-interleukin- 6 receptor antibody in children with systemic-onset juvenile idiopathic arthritis. Arthritis 
Rheum 52: 818-25, 2005.

44. Takei S, Bernstein B, Shaham B, Gallagher K, Reiff A: Safety and Efficacy of high dose Etanercept in Treatment of Juvenile Rheumatoid Arthritis. J Rheumatol 28: 1677-80, 2001.

45. Reiff A, Takei S, Sadeghi S: Etanercept therapy in children with treatment resistant uveitis. Arthritis Rheum 44: 1411-5, 2001 .

46. Smith JR, Levinson RD, Holland GN et al: Differencial eficacy of tumor necrosis factor inhibition in the management of inflamatory eye disease and associated rheumatic disease. Arthritis Rheum 45: 253-7, 2001

47. Smith JA, Thompson DJ, Whitcup SM, Suhlir E, Clarke G, Smith $S$ et al: A randomized, placebo controlled, double masked clinical trial of etanercept for the treatment of uveitis associated with juvenile idiopathic arthritis. Arthritis Rheum 53: 18-23 2005.

48. Prahalad S, Bove KE, Dickens D, Lovell D, Grom A : Etanercept in the treatment of macrophage activation syndrome. J Rheumatol 28: 2120-4, 2001.

49. Bankrust AD: Etanercept and methotrexate combination therapy. Clin Exp Rheumatol 17 (6 supl 18): S69-72, 1999.

50. Schmeling H, Mathony K, John V, Keysser G, Burdach S, Horneff G: A combination of etanercept and methotrexate for the treatment of refractory juvenile idiopathic arthritis: a pilot study. Ann Rheum Dis 60: 410-2, 2001.

51. Bathon JM, Genovesse MC: The early rheumatoid arthritis trial comparing the efficacy and safety of etanercept and methotrexate. Clin Exp Rheumatol, 21 (5 Suppl 31): S 195 -7, 2003.

52. Genovesse MC, Bathon JM, Martin RW et al: Etanercept versus methotrexate in patients with early rheumatoid arthritis: two year radiographic and clinical outcomes. Arthritis Rheum 46: 1443-50, 2002.

53. Gerloni V, Pontikaki I, Desiati F et al: Evaluation of disease activity, disability, and quality of life in patients with persistently active refractory juvenile chronic arthritis after one year treatment with monoclonal anti tumor necrosis factor a antibody (infliximb) Arthritis Rheum 44: S80, 2001.

54. Lahdenne P, Honkanen, V: Infliximab versus etanercept in the treatment of severe juvenile chronic arthritis (JCA). Arthritis Rheum 43(suppl): S381, 2000.

55. Lahdenne P, Vahasalo P, Honkanen V: Infliximab or etanercept in the treatment of children with refractory juvenile idiopathic arthritis: na open label study. Ann Rheum Dis 62: 245-7, 2003.

56. Navarro-Sarabia F, Ariza-Ariza R, Hernandez-Cruz B, Villanueva I: Adalimumab for treating rheumatoid arthritis. Cochrane Database Syst Rev. 20: CD005113, 2005.

57. van de Pute LBA, Atkins $C$, Malaise $M$ et al: Adalimumab (D2E7) monotherapy in the treatment of patients with severely active rheumatoid arthritis. Arthritis Rheum 46(Suppl): S205, 2002

58. Lovell DJ, Ruperto N, Godman S et al: Preliminary data from the study of adalimumab in children with juvenile idiopathic arthritis. Arthritis Rheum 50: S436-7, 2004.

59. Decker L, Ambrust W, Rademaker CM, Prakken B, Kuis W, Wulffraat NM: Safety of anti-TNF therapy in children with juvenile idiopathic arthritis. Clin Exp Rheumatol 22: 252-258, 2004

60. Criscione LG: Tumor necrosis factor alfa antagonists for the treatment of rheumatic diseases. Curr Opin Rheumatol 14:
204-211, 2002.

61. Mikuls TR, Moreland LW: Benefit-risk assessment of infliximab in the treatment of rheumatoid arthritis. Drug Safety 26: 23-32, 2003.

62. Sicotte NL, Voskhull LL: Onset of multiple sclerosis associated with anti TNF therapy. Neurology 57: 1885-8, 2001.

63. Blom BJ: Development of diabetes mellitus during etanercept therapy in child with systemic onset juvenile rheumatoid arthritis. Arthritis Rheum 43: 2068-8, 2000.

64. Favalli EG, Sinigaglia L, Varenna M, Arnoldi C: Drug induces lupus following treatment with infliximab in rheumatoid arthritis. Lupus 359: 753-5, 2002.

65. Shakoor N, Michalska M, Harris CA, Block JA: Drug induced systemic lupus erythematosus associated with etanercept therapy. Lancet 359: 579-80, 2002.

66. Ferracili GF, Assaloni R, Perin A: Drug induced systemic lupus erythematosus and TNF blockers. Lancet 360: 645-6, 2002.

67. Brown SL, Greene MH, Gershon SK, Edwards ET, Braun MM: Tumor necrosis factor antagonist and lymphoma development. Arthritis Rheum 46: 3151-8, 2002.

68. Wolfe F, Michaud K: Lymphoma in rheumatoid arthritis: the effect of metotrexate and anti tumor necrosis factor therapy in 18572 patients. Arthritis Rheum 50: 1703-6, 2004.

69. Smith KJ, Skelton HG: Rapid onset of cutaneous squamous cell carcinoma in patients with rheumatoid arthritis after starting tumor necrosis factor alpha receptor IgG-Fc fusion complex therapy. J Am Acad Dermatol 45: 953-6, 2001.

70. Esses AC, Abril A, Fayne S, Doyle JA: Acute development of multiple keratoacantoma and squamous cell carcinomas after treatment with infliximab. J Am Acad Dermatol 50 (Suppl): S 75-7, 2004.

71. Burge D: Etanercept and squamous cells carcinoma. J Am Acad Dermatol 49(2): 358-9, 2003.

72. Backland G, Nossent H: Acute myeloid leukemia following etanercept therapy. Rheumatology 42: 900-1, 2003.

73. Alcain G, Andrade RJ, Queipo de Lhano MP, Moreno MJ, Garcia-Cortes M, Franquedo E: Acute leukemia after infliximab therapy. Am J gastroenterol 98: 2577, 2003.

74. Ramanan AV, Scheneder R: Macrophage activation syndrome following initiation of etanercept in a child with systemic onset juvenile arthritis. J Rheumatol 30: 401-3, 2003.

75. Kaipiaienen-Seppanen O, Leino M: Recurrent uveitis in a patient with juvenile spondyloarthropathy associated with tumor necrosis factor alfa inhibitors. Ann Rheum Dis 62: 88-9, 2003.

76. Foeldvari I, Krueger E, Scneider T: Acute, non obstructive, steril colecystitis associated with etanercept and infliximab for the treatment of juvenile poliarticular rheumatoid arthritis. Ann Rheum Dis 62: 908-9, 2003.

77. Tutar E, Eriki F, Naçar N, Arici S, Atalay S: Delayed macular, urticarial rash due to infliximab in two children with systemic onset juvenile idiopathic arthritis. Rheumatology 43: 674-5, 2004.

78. Sthepens MC, Shepanski MA, Mamula P, Markowitz JE, Brown KA, Baldassano RN: Safety and steroid sparing experience using infliximab for Chron Disease at a pediatric inflammatory bowel center. Am J Gastroenterol 98: 104-11, 2003.

79. Mohan KA, Cote TR, Siegel JN, Braun MM: Infectious 
complications of biologic treatment of rheumathoid arthritis. Curr Opin Rheumatol 15: 179-84, 2003.

80. Wallis RS, Broder MS, Wong JY, Hanson ME, Beehouwer DO : Granulomatous infections associated with tumor necrosis factor antagonits. Clin Inf Dis 38: 1261-5, 2004.

81. Long R, Gardan M: Tumor necrosis factor inhibitors and the reactivation of latent tuberculosis infection. CMAJ 168: 11536, 2003.

82. Ambrust W, Kamphuis SSM, Wolfs TWF et al: Tuberculosis in a nine years old girl treated with infliximab for systemic juvenile idiopathic arthritis. Rheumatology 43: 527-9, 2004.

83. Ministério da Saúde. Caderno de Atenção Básica - Manual técnico para controle da tuberculose, Brasília, DF, 2002.
84. Mangini C, Melo FAF: Artrite reumatóide, terapia imunossupressora e tuberculose. Rev Bras Reumatol 43: XI$\mathrm{XV}, 2003$.

85. Militão de Albuquerque MFP, Ximenes RAA, Campelo ARL, Sarinho E, Cruz M, Maia Filho V: Neonatal BCG vacine and response to the tuberculin test in BCG vaccinated children in contact with tuberculosis patients in Recife, Brazil. J Trop Pediat 50: 32-6, 2004 .

86. Almeida LMD, Barbieri MA, Da Paixão AC, Cuevas LF: Use of purified protein derivative to asses the risk of infection in children in close contact with adults with tuberculosis in a population with high Calmette Guerin bacilus coverage. Pediat Inf Dis J 20: 1061-5, 2001. 